\title{
DECISION MAKING AT THE LIMITS OF VIABILITY
}

\section{Bührer}

Neonatology, Charité University Medical Center, Berlin, Germany

When the rates mortality and impairment in survivors are close to or exceed $50 \%$, the treatment of extremely immature preterm infants is considered experimental. As such, it requires informed consent by the parents or guardians, as stated by the declaration of Helsinki. In 2010, the International Liaison Committee on Resuscitation, the American Heart Association and the European Resuscitation Council uniformly defined a gestational age of 23 and 24 weeks as the grey zone where parental desires should be supported. This in line with the majority of published national guidelines, but some of these (Austria, Germany) set the grey zone one week lower, while others (Switzerland, France, the Netherlands) one week higher. Published guidelines base thresholds almost universally on gestational age alone. The prognosis of extremely immature infants, however, may be better refined by also taking sex, birth weight, single/multiple status and fetal lung maturation into account. In contrast, presentation of the infant in the delivery room, as evidenced by Apgar scores or acid-base status, has no predictive power for survival or intact survival in extremely preterm infants. Surveys among neonatologists in Europe and North America demonstrate their high propensity to act in a paternalistic fashion for the perceived infant's best interest, while adherence to the principle of informed consent remains low. 